\title{
Efecto local de los colutorios con contenido alcohólico: revisión de la literatura
}

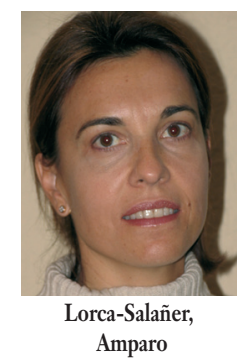

Local effect of alcohol containing mouthwash: review of the literature

Lorca-Salañer, Amparo*
Carrasquer-Burguesa, Assumpła**

*Médico-Odontólogo. Máster en periodoncia.

**Odontólogo. Postgrado en periodoncia.
Resumen: El alcohol ha formado parte de la formulación de enjuagues bucales desde hace más de 50 años. Como otros principios no esta exento de efectos secundarios debidos en su mayor parte al efecto sobre las células epiteliales, por lo que está contraindicada su utilización en pacientes con mucosas lesionadas o sensibles. Recientemente se atribuyeron a los colutorios con alcohol un posible efecto sobre el desarrollo de lesiones de cáncer oral. La revisión de la literatura que disponemos sobre el tema nos muestra que la mayoría de los estudios existentes tienen muchas limitaciones y que no hay indicios de que exista ninguna asociación entre la utilización de colutorios con alcohol y el desarrollo de lesiones cancerígenas. La FDA, la ADA y el NCl aceptan el alcohol como un componente seguro en la formulación de enjuagues.

Palabras clave: Colutorios, Enjuagues, Alcohol.

Abstract: Alcohol has formed part of the formula in mouthwashes for more than 50 years. Like other bases it is not exempt from side effects mainly due to its effects on epithelial cells. This is why its use is contraindicated for patients with epithelial mucosa damage. Recently it has been suggested that alcoholcontaining mouthwash has a possible effect on the development oral cancer lesions. The revision of the available literature on the subject shows us that the majority of the research is very limited and there is no evidence for an existing link between the use of mouthwash with alcohol and the development of cancerogenic lesions. The FDA, the ADA and the $\mathrm{NCl}$ accept alcohol as a safe component of the formula in mouthwashes.

Key words: Mouthwashes, Mouthrinses, Alcohol.

BIBLID [1138-123X (2005)10:4; julio-agosto 369-496]

Lorca-Salañer A, Carrasquer-Burguesa A. Efecto de los colutorios con contenido alcohólico: revisión de la literatura. RCOE 2005;10(4):407-412.
Amparo Locar Salañer

Pérez Pujol, 3. Pta. 2

46002 Valencia

E-mail: amparo.lorca@telefonica.net 


\section{Introducción}

El alcohol forma parte de la formulación de un gran número de enjuagues bucales. Podemos encontrar en el mercado colutorios con disolución alcohólica utilizados por los consumidores desde hace más de 50 años. El alcohol en los colutorios se utiliza como vehículo disolvente de los principios activos de los enjuagues. De forma añadida les aporta sus propiedades antisépticas, además de ser un conservante activo de los componentes de la formulación. Sin embargo, sabemos que al igual que otros productos no esta exento de ciertos efectos secundarios. El alcohol a elevadas concentraciones puede tener efectos lesivos en la mucosa, por lo que no se recomienda en pacientes con alguna patología de base.

Hasta hace poco tiempo la mayoría de enjuagues contenían alcohol, en distintas proporciones. A finales de los años 70 aparecieron publicaciones científicas que sugerían una posible asociación entre el uso diario de enjuagues bucales con alto contenido en alcohol y la aparición de cáncer orofaríngeo y se empezó a cuestionar la seguridad del alcohol como componente de enjuagues bucales. Como medida de precaución, aunque de forma no justificada, la tendencia ha sido a la comercialización de productos que contienen cantidades relativamente bajas de alcohol y a la formulación de colutorios sin alcohol.

Desde la aparición del primer trabajo en 1969 hasta hoy han aparecido gran número de estudios con la finalidad de comprobar o aclarar este tema. Por su parte la ADA (American
Dental Association Council on Dental Therapeutics), la FDA (Food and Drug Administration) junto con el $\mathrm{NCl}$ (Nacional Cáncer Institute) realizaron una exhaustiva revisión. Actualmente con los datos de que disponemos no se ha podido establecer una relación causal entre el uso de colutorios con alcohol y el desarrollo de cáncer oral.

\section{Efectos locales del alcohol sobre la mucosa oral}

El alcohol aplicado de forma tópica tiene un ligero efecto cáustico y astringente por lo que esta contraindicado el uso de colutorios con alcohol en sujetos donde por distintas causas la mucosa oral se encuentra alterada y debido a ello el alcohol que contienen podría lesionarla aún más. Este es el caso de pacientes con mucositis por diversas causas, inmunodeprimidos, irradiados de cabeza y cuello, etc. ${ }^{2 *}$

El alcohol puede provocar una sensación dolorosa que guarda relación con la concentración y la duración del enjuague $^{3}$. No se recomienda el uso de colutorios con alcohol en pacientes que tienen alguna lesión previa en la mucosa. En aquellos sujetos con mucosas sensibles se recomienda diluir el producto las primeras veces y disminuir progresivamente la disolución.

En niños, embarazadas y alcohólicos también esta contraindicada la utilización de colutorios con alcohol, pero los motivos son distintos. En los niños corremos el riesgo de intoxicación accidental ${ }^{4-6}$. Los alcohólicos pueden injerir el colutorio como sustituto de una bebida alcohólica en situaciones desesperadas ${ }^{7}$. También esta contraindicado en pacientes que toman metronidazol y disulfirán por sus efectos intestinales.

Aunque no esta demostrado, se han atribuido al alcohol otras acciones, como la aparición de lesiones de hiperqueratosis, y un efecto erosivo sobre el esmalte y las restauraciones. La relación entre la aparición de lesiones hiperqueratósicas y el uso de colutorios con alcohol, se puso de manifiesto a raíz de un hallazgo de dos casos donde coincidían estas lesiones con el consumo de colutorios con alto contenido en alcohols,. El autor de estos estudios y otros investigadores quisieron demostrar posteriormente esta asociación y diseñaron estudios en animales en los que instalaron una bomba en las mejillas que liberaba de forma continua el producto. En estudios realizados en hámsters necesitaba unas concentraciones elevadísimas durante muchas horas para poder observar algún tipo de lesión en las mucosas. No observaban ningún cambio cuando lo aplicaban un minuto/día. Por ello, no se ha podido demostrar la hipótesis que en principio se planteó.

Respecto a los efectos erosivos en el esmalte y en las restauraciones, disponemos en su mayoría de estudios en laboratorio en los que se observaron sólo cambios microscópicos al sumergir las muestras tiempos prolongados. En estos trabajos no es posible valorar el efecto tampón de la saliva, por lo que las conclusiones no se pueden extrapolar fácilmente. Según los resultados de estudios en laboratorio las alteraciones de las restauraciones se deberían al pH bajo del producto $^{10,11}$. Y parece ser que dependen del tipo material de restauración ${ }^{12}$. 


\section{Colutorios con alcohol y cáncer oral}

Hoy en día hay evidencias de que el consumo crónico de bebidas alcohólicas incrementa de forma moderada el riesgo de desarrollar cáncer en el tracto gastrointestinal superior ${ }^{13}$. El mecanismo patogénico por el cual la ingesta de alcohol promueve el desarrollo del cáncer orofaríngeo no está del todo claro. Se han propuesto distintas hipótesis que tratan de explicar cómo, por vía local y/o sistémica, éste podría actuar como factor de riesgo en el desarrollo de cáncer oral. Hay otro hecho que dificulta el estudio y es la frecuente asociación del alcohol con otras prácticas de riesgo, como el tabaco. Se sabe que el consumo de tabaco incrementa hasta cinco veces el riesgo de padecer cáncer orofaríngeo y este riesgo se incrementa hasta 15 veces cuando alcohol y tabaco actúan conjuntamente.

El hecho de que el alcohol contenido en las bebidas pudiera actuar localmente en la mucosa promoviendo la aparición de lesiones cancerígenas, llevó a pensar a algunos investigadores que el alcohol contenido en los colutorios podría tener un efecto similar y ser también un factor de riesgo en el desarrollo de cáncer oral.

Reflexionando sobre este binomio colutorio-cáncer, parece improbable que exista algún tipo de relación. En primer lugar, aunque no podemos comparar el efecto tópico de una bebida alcohólica con el del alcohol contenido en los colutorios, el efecto de un colutorio con alcohol al 25\% durante 30 segundos podría ser similar al de una o dos bebidas alcohólicas diarias, en ningún caso esto aumenta el riesgo de cáncer oral. Por otro lado,

\begin{tabular}{|lccc|}
\hline Tabla 1. Resumen de los principales estudios con los riesn0s obtenidos \\
\hline Estudios & casos & controles & Riesgo \\
\hline Weaver 1979* & 200 & 50 & $1.14^{*}$ \\
\hline Blot 1983 /1988 & 255 & 502 & 1.04 \\
\hline Winder 1983* & 571 & 571 & $1.26^{*}$ \\
\hline Mashberg 1985 & 95 & 913 & 0.89 \\
\hline Winn 1991* & 866 & 1248 & $1.17^{*}$ \\
\hline Winn 2001 & 342 & 521 & 0.87 \\
\hline Young 1986 & 317 & 306 & 0.75 \\
\hline Kabat 1989 & 125 & 107 & 0.89 \\
\hline Morse 1997 & 127 & 127 & $<0.05$ \\
\hline (*) Estudios en los que se obtienen resultados positivos. \\
\hline
\end{tabular}

aún hoy día, no estamos seguros de que el agente carcinogénico de las bebidas alcohólicas sea el etanol. De hecho el etanol, en sí, no es cancerígeno; lo es el acetaldehído, uno de sus metabolitos. Hay estudios donde se ha comprobado que el efecto de las distintas bebidas alcohólicas sobre la mucosa no es el mismo: el vino tiene menos efecto que la cerveza u otras bebidas ${ }^{14}$

Desde el año 1979 hasta ahora se han publicado numerosos estudios epidemiológicos cuya finalidad ha sido comprobar si existía algún tipo de relación entre el uso habitual de colutorios con alcohol y el desarrollo de cáncer oral. También disponemos de revisiones recientes sobre la literatura publicada, como la de Gagari ${ }^{15}$, Elmo$\mathrm{re}^{16}$, cole $\mathrm{e}^{17^{* *}}$ y Carretero ${ }^{2}$

Estos cuatro autores revisaron los estudios epidemiológicos donde se estudia la posible influencia del uso de colutorios en el desarrollo del cáncer orofaríngeo. Los artículos revisados son bastante similares y finalmente llegan a las mismas conclusiones: en primer lugar, los artículos disponibles tienen muchas deficiencias en sus diseños y realización, y en segundo lugar, a pesar de ello, sólo existen tres donde se encuentran resultados positivos en un grupo de pacientes (tabla 1). Finalmente concluyeron que no existe ningún dato que pueda atribuir al uso continuado de colutorios que contienen alcohol en su formulación, algún efecto en la aparición de cáncer orofaríngeo.

Por su parte la ADA, la FDA y el NCl, revisando de forma separada los trabajos realizados llegan a resultados similares: coinciden tanto en las deficiencias que presentan la mayoría de estudios que seguidamente analizaremos, como en la conclusión de que con los datos disponibles en el momento no se puede establecer una relación causal entre el uso de enjuagues bucales con contenido en alcohol y el cáncer oral.

Antes de pasar a analizar de forma separada los resultados de los estudios epidemiológicos vamos a realizar una serie de consideraciones en cuanto a las limitaciones $\mathrm{y} / \mathrm{o}$ deficiencias encontradas en ellos: 


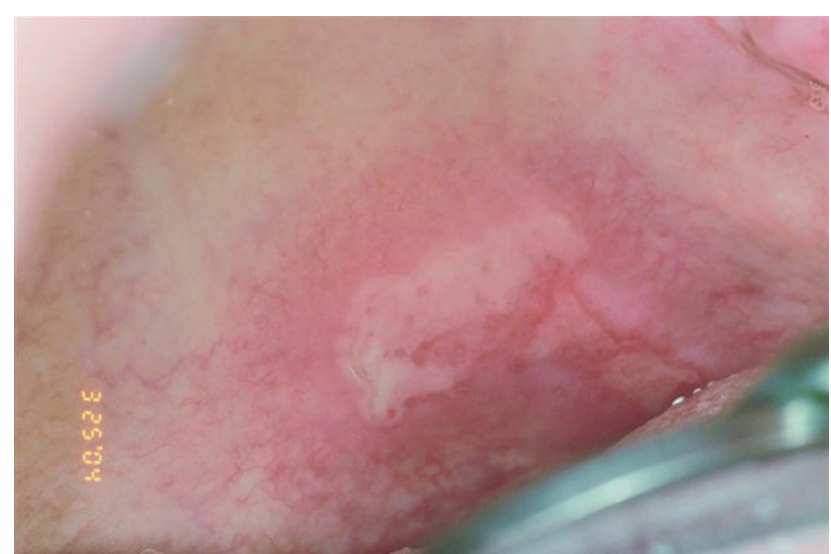

Figura 1. Penfigoide en mucosa palatina. Este tipo de lesiones contraidica el tratamiento con colutorios con alcohol.

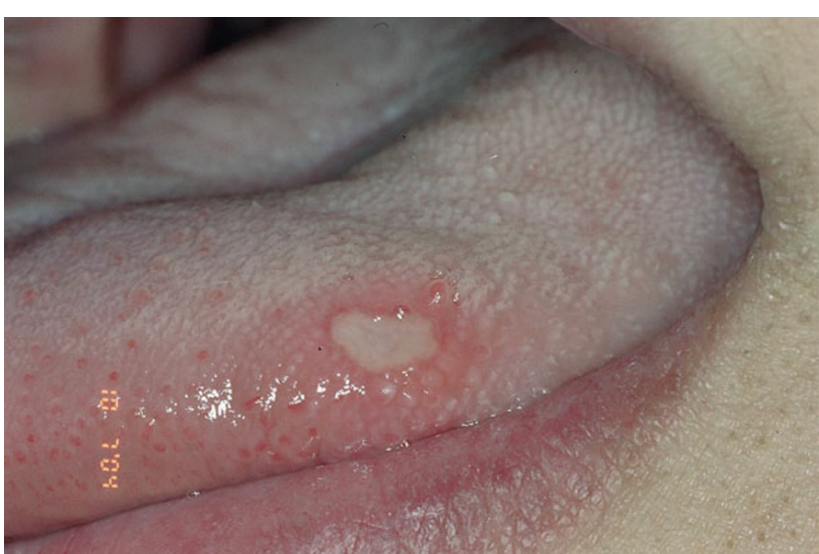

Figura 2. Fotografía de un afta. La realización de enjuagues con alcohol puede provocar dolor
- En primer lugar, las muestras tomadas para la mayoría de los estudios fueron poblaciones hospitalarias con patologías.

- Todos los estudios se realizaron en enfermos de cáncer oro faríngeo sin distinguir la topología del cáncer ni la localización. Faltan historias médicas y documentación sobre los casos.

- Se incluyeron como patología casos de cáncer en tejidos probablemente sin contacto directo con los enjuagues bucales. La mayoría de estudios tomaron como casos poblaciones con cáncer orofaríngeo incluyendo en algunos el cáncer de cabeza y cuello.

- Para poder valorar el efecto del colutorio deberíamos de eliminar el efecto del alcohol y el tabaco, que sabemos que son factores de riesgo probados. Esto resulta muy difícil ya que casi todos los enfermos de cáncer orofaríngeo tienen presente alguno de estos dos factores.

- No existen bases científicas ni biológicas que expliquen los distintos resultados obtenidos entre hombres y mujeres.

- La mayoría de los trabajos no tienen datos acerca de la concentración de alcohol de los colutorios ni sobre el tiempo de empleo.

- Todos ellos son estudios retrospectivos donde los datos sobre alcohol, tabaco y uso de colutorios se obtienen a través de un cuestionario, incluso en alguno de ellos por encuesta telefónica.

- Hay que tener en cuenta que es difícil controlar a los individuos que consumen alcohol en exceso, ya que no suelen reflejar las cifras reales de exposición al alcohol; lo mismo se puede decir del tabaco. Con el uso de colutorios, sin embargo, no existen presiones sociales que conduzcan a falsear la información.

Aún con todas las deficiencias encontradas, los hallazgos de los estudios son inconsistentes.

\section{Revisión de los estudios epidemiológicos}

De los diez estudios epidemiológicos diseñados para valorar la relación entre la utilización de colutorios con alcohol a largo plazo y la existencia de lesiones de cáncer oral sólo en tres se obtuvieron resultados positivos (tabla 1).
El primer estudio que se realizó fue el de Weaver en $1979^{18}$. Este autor, en una serie de 200 pacientes con cáncer de células escamosas de cabeza y cuello, identificó a 11 individuos que no fumaban ni bebían. Diez de estos utilizaban habitualmente un colutorio y 9 de ellos usaban un colutorio con más de un $25 \%$ de alcohol en su composición. Aunque globalmente los resultados no eran significativos y sólo eran positivos para estos 11 pacientes, el autor establece una posible relación causal entre ambos factores.

Como respuesta al estudio de Weaver, en 1983 Blot y sus colaboradores $^{19}$, se pusieron en contacto de nuevo con sujetos de un estudio anterior realizado en mujeres que presentaban cáncer orofaríngeo para estudiar en este momento el efecto de los colutorios, ya que en la investigación anterior se estudió sólo el tabaco y el alcohol. Se pusieron en contacto de nuevo con los pacientes a través de encuesta telefónica; consiguieron hablar con 206 casos de los 237 iniciales y con 352 de los 502 controles. Los autores no encontra- 
ron ninguna asociación entre colutorios y cáncer orofaríngeo en las mujeres fumadoras. Sin embargo, aunque no fue significativo, se obtuvo un mayor riesgo en mujeres no fumadoras. No obtuvieron resultados consistentes en cuanto a la relación dosis-respuesta.

En el año 1983 Wynder realizó un estudio sobre 571 pacientes hospitalizados que padecían cáncer orofaríngeo y tomó 571 controles. Analizando la relación entre el uso de colutorios y la presencia de cáncer orofaríngeo, los resultados fueron negativos para el grupo de hombres. Sin embargo apareció una relación moderada en el caso de las mujeres. No se disponía información acerca del contenido de alcohol de los productos ni sobre la causa por la cual los utilizaban. No apareció relación con el tiempo que llevaban realizando enjuagues ${ }^{20}$.

En 1985, Mashberg valoró la relación entre el uso de colutorios con alcohol y el cáncer orofaríngeo en 95 hombres que padecían algún cáncer de este tipo. Tomo 913 pacientes, escogidos dentro del mismo hospital, tanto los casos, como controles. El análisis de los resultados no demostró diferencias estadísticamente significativas entre casos y controles en cuanto a la frecuencia con la que realizaban los enjuagues. No aparecieron diferencias tampoco al relacionar el uso de colutorios con la edad, tabaquismo y alcoholismo. Los autores concluyeron que no existía evidencia de que los enjuagues bucales con alcohol fueran un factor de riesgo en el cáncer oral y faríngeo en hombres $^{21}$.

En 1986, Young realizó un estudio multihospitalario de diseño caso-con- trol, para investigar los factores de riesgo en hombres y mujeres con cáncer orofaríngeo. Entre otros factores, se estudió el alcohol, el tabaco, los colutorios, la higiene oral y la ocupación. Se tomaron 317 casos de cáncer orofaríngeo y se utilizaron como controles 306 sujetos. No apareció asociación con el uso de colutorios y la existencia de cáncer oral, orofaríngeo e hipofaríngeo ${ }^{22}$.

En 1989, Kabat retomó el estudio de Wynder en el que obtenían resultados positivos en mujeres. Centrándose en este grupo, Kabat observó una relación moderada entre el uso de colutorios y cáncer orofaríngeo en el caso de mujeres que utilizaban el colutorio para eliminar la halitosis producida por el tabaco y el alcohol. Por lo que en sus conclusiones, Kabat anotó que para el diseño de futuros estudios, no sólo hay que registrar el uso de colutorios sino que también, es importante averiguar la causa por la que se utilizan los colutorios ${ }^{23}$.

En 1997, Morse y sus colaboradores realizaron un estudio caso-control con el objetivo de estudiar aquellos posibles factores de riesgo, entre ellos el uso de colutorios, que podían intervenir en la aparición de lesiones de displasia epitelial. Analizaron 127 casos y tomaron 127 controles. Los individuos con displasia epitelial tienen un riesgo mayor de desarrollar cáncer oro-faríngeo. Los resultados de este estudio no mostraron relación entre el uso de enjuagues y la displasia epitelial oral24.

Winn, en 2001, realizó un estudio sobre 342 casos y 521 controles en enfermos de cáncer orofaríngeo en Puerto Rico. Valoraron el efecto del alcohol, el tabaco y el uso de coluto- rios. Los autores no encontraron asociación entre la aparición de cáncer orofaríngeo y el uso habitual de colutorios con alcohol. No apareció relación dosis-respuesta. Los resultados fueron positivos en no fumadores que habían sido alcohólicos. Estos mismos autores tienen otro trabajo anterior, publicado en 1991, donde observaron resultados positivos entre el uso de colutorios y la aparición de cáncer orofaríngeo en mujeres. En este estudio, sin embargo, el efecto de los colutorios se observaba solo en los grupos que bebían y/o fumaban, mientras que no aparecían resultados positivos en aquellos casos que ni bebían ni fumaban. Estos resultados no dejan claro si es el propio colutorio el factor o son factores relacionados con su uso la causa de ellos ${ }^{25,26}$.

En alguno de estos trabajos aparece una asociación entre el uso de colutorios y la aparición de lesiones cancerígenas en poblaciones de mujeres. Podría ser que el uso de colutorios se correlacione con algún factor de riesgo desconocido entre personas que no consumen alcohol ni tabaco. Puede ser también que los pacientes utilizasen los colutorios para tratar los primeros síntomas de la patología y no sean la causa en sí mis$\mathrm{ma}^{17 *, 27}$

Podemos decir que actualmente hay datos suficientes que implican a los siguientes factores en la etiología del cáncer oral: el tabaco, el excesivo consumo de bebidas alcohólicas, la mala higiene oral y las ausencias dentarias con falta de reposición de los mismos. Incluso algún autor que habla de una predisposición genética y otros que apuntan la posibilidad de 
que factores de la dieta actúen también como factores de riesgo. Pero a la vista de los resultados de estos estudios, podemos decir que, no existen datos que nos permitan establecer alguna relación entre el uso de colutorios con alcohol y la aparición de lesiones de cáncer oral.

\section{Conclusión}

Hoy por hoy no se ha podido establecer una relación causal entre el uso de colutorios y el desarrollo de cáncer oral. Por lo tanto, los dentistas generales pueden recomendar a sus pacientes el colutorio que consideren oportuno incluyendo aquellos que contienen alcohol en su composición. Úni-camente estaría contraindicado su uso en niños, embarazadas, alcohólicos y en pacientes con patología erosiva y susceptible, como mucositis, irradiados de cabeza y cuello, e inmunodeprimidos.

\section{Bibliografla recomendada}

Para profundizar en la lectura de este tema, el/los autor/es considera/an interesantes los artículos que aparecen señalados del siguiente modo: *de interés ${ }^{*}$ de especial interés.

1. Wu CD, Savitt E. Evaluación de la seguridad y la eficacia de los productos de higiene oral de venta sin receta en la reducción y el control de placa y la gingivitis. Periodontology 2000. 2003:3:91-105.

2.** Carretero-Pelaéz Ma A, Esparza-Gómez GC, Figuero-Ruiz E, Cerero Lapiedra R. Colutorios con alcohol y su relación con el cáncer oral. Análisis crítico de la literatura. Med Oral 2004;9:116-23.

Se trata de una revisión de la literatura especialmente interesante por el método estadístico que utiliza para poder comparar los estudios, analizando el Odds ratio y el índice de asociación.

3. Bolanowski SJ, Gescheider GA, Sutton SVW. Relationship between oral pain and ethanol concentration in mouthrinses. J Periodont Res 1995;30:192-7.

4. Westermeyer RR, Terpolilli RN. Cardiac asystole after mouthwash ingestion: a case report an review of the contents. Mil Med 2001;166: 833-5.

5. Shulman JD, Wells LM. Acute ethanol toxicity from ingesting mouthwash in children younger than 6 years of age. Pediatr Dent 1997;19: 404-8.

6. Hornfeldt CS. A report of acute ethanol poisoning in a child: Mouthwash, cologne, perfume and after-shave. J Toxicol Clin Toxicol 1992;30:115-21.

7. Sperry K, Pfalzgraf R. Fatal ethanol intoxication from household products not intention for ingestion. J Forensic Sci 1990:35:1138-42.

8. Bernstein ML. Oral Mucosal white lesions associated with excessive use of Listerine mouthwash: report of two cases. Oral Surg Oral Med Oral Pathol 1978;46:781-5.

9. Bernstein ML, Carlish R. The induction of hiperkeratotic white lesions in hamster cheek pouches with mouthwash. Oral Surg 1979; 48:517-22.

10. Pretty IA, Edgar WM, Higham SM. The erosive potential of commercially available mouthwashes on enamel as measured by quantitative light-induced by fluorescence (QLF). J Dent 2003 Jul;31:313-9

11. Penugonda B, Settembrini L, Scherer W, Hittelman E, Strassle. Alcohol- containing mouthwashes: effect on composite resin hardness. J Clin Dent 1994;5:60-2.

12. Yap AUJ, Tan BWY, Tay LC, Chang KM, Loy TK y Mok BYY. Effect of mouthrinses on microhardness and wear of composite and componer restoratives. Operative Dentristy 2003;28:740-6.

13. Jensen O, Paine S, McMichael A, Ewertz M. Alcohol. In: Schotten-feld D, Fraumeni J, eds. Cancer epidemiology and prevention. 2nd ed. New York : Oxford University Press, 1996.

14. Gronbaek M, Becker U, Johansen D, Tonnesen H,Jensen G, Sorensen T. Population based cohort study of the association between alcohol intake and cancer of the upper digestive tract. BMJ 1998:317:844-8.

15. Gagari E,Kabani S. Adverse Effect of Mouthwash Use. A review. Oral Surg Oral Med Oral Pathol Oral Radiol Endod 1995;80:432-9.

16. Elmore JG, Horwitz RI. Oral cancer and mouthwash use: Evaluation of the epidemiologig evidence. Otolaryngol Head Neck Surg 1995;113:253-61

17.** Cole P, Rodu B, Mathisen A. Alcohol-containing mouthwash and oropharyngeal cancer: a review of the epidemiology. J Am Dent Assoc 2003;134:1079-87.
Realiza un revisión profunda de los estudios epidemiológicos relacionados con el tema. Detallando las limitaciones que encuentran en ellos y análisis de resultados.

17. Weaver A, Fleming S, Smith D. Mouthwash and oral cancer: carcinogen or coincidence? J Oral Surg 1979;37:250-3.

18. Blot W, Winn D, Fraumeni J. Oral cancer and mouthwash. J Natl cancer Inst 1983;70:251-3.

19. Wynder E, Kabat G,Rosenberg S, Levenstein M. Oral cancer and Mouthwash use. J Natl cancer Inst 1983;70:255-70.

20. Mashberg A, Barsa P, Grossman M. A study of the relationship between mouthwash use and oral and pharyngeal cancer. J Am Dent Assoc 1985;110:731-4.

21. Young TB, Ford CN, Brandenburg JH. An epidemiologic study of oral cancer in a statewide netwoek. Am J Otolaringool 1986;7:200-8.

22. Kabat G, Hebert J, Wynder E. Risk factors of oral cancer in women. Cancer Res 1989;49: 2803-6.

23. Morse D, Katz R, Pendrys D et al. Mouthwash use and dentures in relation to oral apithelial dysplasia. Oral Oncol 1997;33:338-43.

24. Winn D, Blot W, Shy C, Pickle L, Toledo A, Fraumeni J. Snuff dipping and oral cancer among women in the southern United States. New Engl J Med 1981;304:745-9.

25. Winn D,BlotW, McLaughlin J et al. Mouthwash use and oral conditions in the risk of oral and pharyngeal cancer. Cancer Res 1991; 51:3044-7.

26. Marsall JR, Graham S, Haughey BP, Shedd D, O'Shea R, Brasure J et al. Smoking, Alcohol, Dentition and Diet in the Epidemiology of Oral Cancer. Oral Oncology Eur J Cancer 1992;28:9-15. 